\title{
Persistência de óleos essenciais em milho armazenado, submetido à infestação de gorgulho do milho
}

\author{
Persistence of essential oils in stored maize submitted to infestation of maize weevil
}

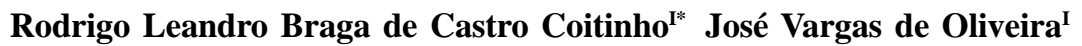 \\ Manoel Guedes Corrêa Gondim Júnior ${ }^{\mathrm{I}}$ Cláudio Augusto Gomes da Câmara ${ }^{\text {II }}$
}

\section{RESUMO}

Os óleos essenciais e os compostos constituintes têm sido pesquisados quanto a sua atividade inseticida contra pragas de grãos armazenados. Neste trabalho, avaliou-se a persistência de óleos essenciais em milho armazenado, submetido à infestação do gorgulho do milho, Sitophilus zeamais Mots. (Coleoptera: Curculionidae). A persistência dos óleos e do eugenol foi avaliada no período inicial (logo após a impregnação) e aos 30, 60, 90 e 120 dias de armazenamento. As mortalidades de S. zeamais, no período inicial, variaram entre 93,8 (Piper hispidinervum, Melaleuca leucadendron e eugenol) e 100\% (Eugenia uniflora, frutos verdes de Schinus terebinthifolius e Piper marginatum). A partir dos 30 dias, as mortalidades, de modo geral, decresceram, com exceção de P. marginatum (92,2\%), que alcançou 53,1\% de mortalidade aos 120 dias de armazenamento. De acordo com as equações de regressão ajustadas para o número de $\boldsymbol{S}$. zeamais emergidos em todo o período de armazenamento, apenas não houve significância para os óleos de S. terebinthifolius, P. marginatum e testemunha. Em relação à média geral, o óleo de $\boldsymbol{P}$. marginatum foi o mais persistente, proporcionando emergência de apenas 0,30 insetos, diferindo dos óleos restantes, do eugenol e da testemunha. Os demais tratamentos só diferiram em relação à testemunha.

Palavras-chave: atividade inseticida, efeito residual, gorgulho do milho, óleos essenciais.

\section{ABSTRACT}

The essential oils and constituent compounds have been studied for their insecticidal activity against stored grain pests. In this research, persistence of the essential oils in stored maize subject to infestation by maize weevil, Sitophilus zeamais Mots. (Coleoptera: Curculionidae) were evaluated. Persistence of oils and eugenol were evaluated in the initial period (after impregnation) and at 30, 60, 90 and 120 days of storage. Mortalities of $\mathbf{S}$. zeamais in the initial period ranged between 93.8 (Piper hispidinervum, Melaleuca leucadendron and eugenol) to $100 \%$ (Eugenia uniflora, green fruits of Schinus terebinthifolius and Piper marginatum). From the 30th day, mortalities declined, except for P. marginatum (92.2\%), which reached $53.1 \%$ at 120 days of storage. According to the regression equations adjusted for the number of $\boldsymbol{S}$. zeamais emerged throughout the storage period, there was no significance for the oils of $\boldsymbol{S}$. terebinthifolius, $\boldsymbol{P}$. marginatum and the control. Considering general average, the oil of $\boldsymbol{P}$. marginatum was the most persistent, providing emergency of 0.30 insects; differing from the other oils, as eugenol and the control. The other treatments differed in relation to the control.

Key words: insecticidal activity, residual effect, maize weevil, essential oils.

\section{INTRODUÇÃO}

A preocupação dos consumidores em relação ao ambiente, à qualidade do alimento e aos efeitos colaterais dos inseticidas sintéticos têm incentivado os pesquisadores a testar alternativas para o controle de pragas de grãos armazenados, como os óleos essenciais obtidos de plantas e seus compostos constituintes (MOREIRA et al., 2007; RAJENDRAN \& SRIRANJINI, 2008). Esses produtos, geralmente,

IDepartamento de Agronomia, Universidade Federal de Pernambuco (UFRPE), 52171-900, Recife, PE, Brasil. E-mail: rodrigolbc@bol.com.br. *Autor para correspondência.

ID Departamento de Química, UFRPE, Recife, PE, Brasil. 
são de baixa toxicidade para o homem e os animais, custo relativamente baixo, degradam-se rapidamente no ambiente e não contaminam os alimentos com resíduos tóxicos (ISMAN, 2006). Atuam nos insetos por contato, ingestão e fumigação (KETOH et al., 2006; SAHAF \& MOHARRAMIPOUR, 2008) e provocam diversos efeitos nesses artrópodes, como mortalidade, repelência, deterrência na alimentação e na oviposição, redução no crescimento e deformações (ISMAN, 2006).

Estudos sobre a persistência de óleos essenciais na proteção de sementes e grãos contra o ataque de pragas são, de um modo geral, escassos, mas indicam que estes se degradam rapidamente (ISMAN, 2006). A persistência dos óleos depende da sua volatibilidade, da toxicidade e do tipo de óleo (essencial ou fixo). Estudos desenvolvidos com milho armazenado e infestado com Sitophilus zeamais Mots. (Coleoptera: Curculionidae) têm demonstrado que, na maioria dos casos, a mortalidade decresce com o período de armazenamento e o número de insetos emergidos aumenta, em decorrência da redução da sua persistência (COITINHO et al., 2006; ASAWALAM \& HASSANALI, 2006).

A importância de pesquisas com métodos alternativos aos inseticidas químicos sintéticos, que se enquadrem nos princípios do manejo de pragas de grãos armazenados, é de grande utilidade, principalmente para os pequenos e médios produtores de milho, visando não apenas controlar infestações de $\boldsymbol{S}$. zeamais, mas também preservar a saúde dos consumidores da presença de resíduos tóxicos. Assim, o presente trabalho objetivou avaliar a persistência de óleos essenciais e o composto eugenol em sementes de milho infestadas com gorgulho do milho, S. zeamais.

\section{MATERIAL E MÉTODOS}

O presente trabalho foi desenvolvido no Laboratório de Entomologia Agrícola do Departamento de Agronomia, Área de Fitossanidade, e no Laboratório de Produtos Naturais Bioativos do Departamento de Química da Universidade Federal Rural de Pernambuco (UFRPE), em Recife, Pernambuco (PE).

Criação de $\boldsymbol{S}$. zeamais. Os insetos foram criados à temperatura de $28,7 \pm 1,12^{\circ} \mathrm{C}$, umidade relativa de $61,3 \pm 2,6 \%$ e fotofase de 12 horas, em sementes de milho da cultivar 'Caatingueiro', acondicionadas em recipientes de vidro fechados com tampa plástica perfurada e revestida internamente com tecido fino para permitir as trocas gasosas. O confinamento dos insetos foi realizado durante 15 dias para efetuarem a postura e, em seguida, estes foram retirados, e os recipientes foram estocados até a emergência da geração $\mathrm{F}_{1}$. Efetuou-se esse procedimento por sucessivas gerações, de modo a assegurar a quantidade de adultos necessários para a execução dos experimentos.

Coleta do Material Vegetal. Folhas e frutos de Eugenia uniflora L. (Myrtaceae), Cinnamomum zeylanicum Blume (Lauraceae), Schinus terebinthifolius Raddi. (Anacardiaceae), Melaleuca leucadendron L. (Myrtaceae) e Piper marginatum Jacq. (Piperaceae) foram coletados em Recife e Olinda, PE. O material foi identificado pela professora Margareth Ferreira de Sales do Departamento de Biologia da UFRPE, e as exsicatas encontram-se depositadas no Herbário Vasconcelos Sobrinho da UFRPE, sob os números 48490, 48602, 49259, 48489 e 48210, respectivamente.

Obtenção e Composição dos Óleos Essenciais. O material vegetal coletado foi submetido à hidrodestilação por $2 \mathrm{~h}$, no Laboratório de Produtos Naturais Bioativos do Departamento de Química da UFRPE, e os óleos essenciais foram extraídos por meio de um aparelho tipo Clevenger modificado, separados da água, secos com $\mathrm{Na}_{2} \mathrm{SO}_{4}$ anidro e armazenados à baixa temperatura, em recipientes escuros hermeticamente fechados. O rendimento dos óleos foi calculado com base no peso do material fresco. Também foram utilizados o composto eugenol e os óleos essenciais de Piper aduncum L. (Piperaceae) e Piper hispidinervum C.DC. (Piperaceae) obtidos da Indústria Farmacêutica Ltda, Paraná (PR), da Comissão Executiva do Plano da Lavoura Cacaueira (CEPLAC PA) e da Embrapa - Acre, respectivamente.

Os resultados das análises dos óleos essenciais das plantas coletadas em Recife e Olinda foram cedidos por CÂMARA (2008 - Informe verbal), constando dos seguintes compostos majoritários: folhas de $\boldsymbol{E}$. uniflora - Epóxido de selina - 1,3,7, (11)trien- 8 ona $-10,6 \%$; folhas de C. zeylanicum - Benzoato de benzila $-64,4 \%$; folhas/frutos de $S$. terebinthifolius -p-Cimen-7-ol-22,5\% e 9-epi-(E)-Cariofileno-10,1\%; folhas de M. lencadendron-(E)-Nerodidol-92,5\%; $\boldsymbol{P}$. marginatum - (Z)-Asarona-30,5\%.

Os compostos majoritários dos óleos essenciais de folhas de P. aduncum e P. hispidinervum constam, respectivamente, dos trabalhos de FAZOLIN et al. (2007) e MAIA et al. (1998). O composto eugenol não foi analisado, sendo utilizado em razão de estudos comprobatórios da atividade inseticida com insetos de grãos armazenados (COITINHO et al., 2006).

Eliminação da Infestação e Equilíbrio da Umidade das Sementes. Sementes de milho foram 
acondicionadas em sacos plásticos e mantidas em freezer sob temperatura de $-10^{\circ} \mathrm{C}$, durante sete dias, para eliminação de eventuais infestações de insetos provenientes do campo. Após a retirada do freezer, foram transferidas para frascos de vidro, mantidos no laboratório à temperatura ambiente durante 10 dias, com a finalidade de atingirem o equilíbrio higroscópico.

Persistência de Óleos Essenciais em Milho Armazenado. Os experimentos foram conduzidos à temperatura de $27,4 \pm 0,91^{\circ} \mathrm{C}$, umidade relativa de $62,5 \pm 2,33 \%$ e fotofase de 12 horas. Foram utilizados os seguintes tratamentos nas concentrações, em $\mu \mathrm{L}$ $40 \mathrm{~g}^{-1}$ de milho: folhas de P. hispidinervum $(2,8)$, eugenol (19,0), folhas de $\boldsymbol{P}$. marginatum $(40,0)$, frutos verdes de $\boldsymbol{S}$. terebinthifolius $(125,0)$, folhas de $\boldsymbol{S}$. terebinthifolius $(80,0)$, folhas de $\boldsymbol{E}$. uniflora $(35,0)$, folhas de $\boldsymbol{M}$. leucadendron (120,0), folhas de $\boldsymbol{C}$. zeylanicum $(30,0)$ e testemunha. As concentrações foram escolhidas em testes preliminares. Os óleos foram misturados às sementes no interior de recipientes de vidro, com o auxílio de pipetador automático, os quais foram agitados manualmente durante dois minutos. A parcela foi constituída de $40 \mathrm{~g}$ de sementes de milho da cultivar 'Caatingueiro' acondicionados em recipiente de vidro fechado com tampa plástica perfurada e revestida internamente com tecido fino para permitir as trocas gasosas. Cada recipiente foi infestado com 16 adultos não sexados de $\boldsymbol{S}$. zeamais, com zero a 15 dias de idade (COITINHO et al., 2006). Decorridos sete dias do confinamento, efetuou-se a contagem dos insetos vivos e mortos, descartando-os em seguida. Os adultos emergidos foram quantificados, diariamente, e descartados a partir do $35^{\circ}$ dia do confinamento até o término da emergência (cinco dias consecutivos sem emergência). Os experimentos foram realizados em cinco períodos de armazenamento: período inicial (logo após a impregnação - T0), um mês (T1), dois meses (T2), três meses (T3) e quatro meses (T4), no delineamento experimental inteiramente casualizado, constando de nove tratamentos, cinco e quatro repetições.

Os resultados de mortalidade (\%) e de adultos emergidos foram submetidos aos testes de normalidade e homogeneidade de variância. A porcentagem de mortalidade foi transformada em arco seno $(\mathrm{x} / 100)^{0,5}$, e o número de adultos emergidos, em $(\mathrm{x}+1)^{0,5}$, para atender os requisitos da análise de variância (ANOVA). Em seguida, os resultados foram submetidos à ANOVA, com medidas repetidas no tempo, sendo considerados os nove tratamentos (óleos e testemunha) e as avaliações no tempo como medidas repetidas. De acordo com os resultados da
ANOVA, quando significativas para tratamento, as médias entre tratamentos foram separadas usando-se o teste de Tukey HSD $(\mathrm{P}<0,05)$ e, quando significativas no tempo (avaliações), estas foram submetidas à análise de regressão para cada óleo, sendo selecionado o modelo que melhor se ajustou aos dados. Todas as análises foram realizadas utilizando-se o programa computacional SAS, versão 9.00 (SAS INSTITUTE, 2002).

\section{RESULTADOS E DISCUSSÃO}

Persistência de Óleos Essenciais em Milho Armazenado. No período inicial de armazenamento, os óleos essenciais e eugenol não diferiram estatisticamente $(\mathrm{P}>0,05)$. As mortalidades variaram entre 93,8 (folhas de $\boldsymbol{P}$. hispidinervum, folhas de $\boldsymbol{M}$. leucadendron e eugenol) e 100\% (folhas de $\boldsymbol{E}$. uniflora, frutos verdes de S. terebinthifolius e folhas de $\boldsymbol{P}$. marginatum). A partir dos 30 dias, as mortalidades, de um modo geral, decresceram, com exceção de $\boldsymbol{P}$. marginatum, que provocou mortalidades, respectivamente, de 92,2; 89,1; 93,8 e $53,1 \%$, aos 30, 60, 90 e 120 dias de armazenamento (Tabela 1). De acordo com as equações de regressão ajustadas para o número de $\boldsymbol{S}$. zeamais emergidos em todo o período de armazenamento, apenas não houve significância para os óleos de folhas de $\boldsymbol{S}$. terebinthifolius e de P. marginatum e testemunha. Em relação à média geral, o óleo de $\boldsymbol{P}$. marginatum foi o mais persistente, proporcionando emergência de apenas 0,30 insetos, diferindo dos óleos restantes, do eugenol e da testemunha. Os demais tratamentos só diferiram da testemunha (Tabela 2).

De um modo geral, o decréscimo na persistência dos óleos essenciais com o prolongamento do período de armazenamento do milho parece ter sido decorrente das diferenças de volatilidade destes, resultando em uma persistência limitada, conforme observações de ISMAN (2006). COITINHO et al. (2006) constataram que, no período inicial de armazenamento (logo após a impregnação), os óleos de Eucalyptus globulus Labill (Myrtaceae), o composto eugenol, Lippia gracillis HBK. (Verbenaceae), Azadirachta indica A. Juss (Meliaceae) e Caryocar brasiliense Camb. (Caryocareceae) provocaram 100,0\% de mortalidade de adultos de S. zeamais; porém, aos 60 e 120 dias de armazenamento, a mortalidade foi inexpressiva. Por outro lado, os óleos fixos de girassol, Helianthus annuus L. (Asteraceae), milho, Zea mays L. (Poaceae), oliva, Olea europaea L. (Oleaceae), e soja, Glycine $\max ($ L.) Merr. (Fabaceae), na concentração de 
Tabela 1 - Porcentagens de mortalidade (média \pm EP) de Sitophilus zeamais em sementes de milho tratadas com óleos essenciais, em diferentes concentrações, após a impregnação e aos 30, 60, 90 e 120 dias de armazenamento. Temp: $27,4 \pm 0,91^{\circ} \mathrm{C}, \mathrm{UR}$ : $62,5 \pm 2,33 \%$ e fotofase de $12 \mathrm{~h}$.

\begin{tabular}{|c|c|c|c|c|c|c|}
\hline \multirow{2}{*}{ Tratamento } & \multirow{2}{*}[]{$^{3} \mu \mathrm{L} 40 \mathrm{~g}^{-1}$} & \multicolumn{5}{|c|}{ 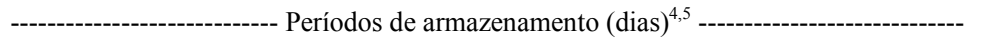 } \\
\hline & & 0 & 30 & 60 & 90 & 120 \\
\hline Piper hispidinervum ${ }^{1}$ & 2,8 & $93,8 \pm 2,55 \mathrm{a}$ & $14,1 \pm 2,99 b$ & $4,7 \pm 2,99 b$ & $4,7 \pm 2,99 \mathrm{bc}$ & - \\
\hline Melaleuca leucadendron ${ }^{1}$ & 120,0 & $93,8 \pm 2,55 \mathrm{a}$ & $1,6 \pm 1,56 \mathrm{~d}$ & - & $1,6 \pm 1,56 \mathrm{c}$ & - \\
\hline Eugenol & 19,0 & $93,8 \pm 2,55 \mathrm{a}$ & $10,9 \pm 1,56 \mathrm{bc}$ & $3,1 \pm 1,80 \mathrm{~b}$ & $3,1 \pm 3,13 \mathrm{bc}$ & $1,6 \pm 1,56 \mathrm{~b}$ \\
\hline $\begin{array}{l}\text { Cinnamomum } \\
\text { zeylanicum }\end{array}$ & 30,0 & $96,9 \pm 1,80 \mathrm{a}$ & $5,0 \pm 0,20 \mathrm{~cd}$ & $3,1 \pm 1,80 \mathrm{~b}$ & $1,6 \pm 1,56 \mathrm{c}$ & - \\
\hline Schinus terebinthifolius ${ }^{2}$ & 125,0 & $100,0 \pm 0,00 \mathrm{a}$ & $4,7 \pm 2,99 \mathrm{~cd}$ & $9,4 \pm 4,03 b$ & $15,6 \pm 3,13 b$ & - \\
\hline Eugenia uniflora ${ }^{1}$ & 35,0 & $100,0 \pm 0,00 \mathrm{a}$ & $6,3 \pm 0,00 \mathrm{bcd}$ & $4,7 \pm 2,99 b$ & $6,3 \pm 2,55 \mathrm{bc}$ & $4,7 \pm 4,69 b$ \\
\hline Piper marginatum ${ }^{1}$ & 40,0 & $100,0 \pm 0,00 \mathrm{a}$ & $92,2 \pm 1,56 \mathrm{a}$ & $89,1 \pm 8,98 \mathrm{a}$ & $93,8 \pm 4,42 \mathrm{a}$ & $53,1 \pm 4,03 \mathrm{a}$ \\
\hline Schinus terebinthifolius ${ }^{1}$ & 80,0 & $98,4 \pm 1,56 \mathrm{a}$ & $3,1 \pm 1,80 \mathrm{~cd}$ & - & $10,9 \pm 3,93 \mathrm{bc}$ & - \\
\hline Testemunha & 0,0 & $-{ }^{6}$ & - & - & - & - \\
\hline
\end{tabular}

${ }^{1}$ Folhas; ${ }^{2}$ Frutos Verdes; ${ }^{3}$ Concentração; ${ }^{4}$ Médias seguidas de mesma letra na vertical não diferem significativamente entre si pelo teste de Tukey HSD $(\mathrm{P}>0,05) ;{ }^{5}$ Dados transformados em arco seno $(\mathrm{x} / 100)^{0,5} ;{ }^{6}$ Não houve mortalidade.

$5 \mathrm{~mL} \mathrm{~kg}{ }^{-1}$ de grãos de milho, ocasionaram, respectivamente, 53,$2 ; 56,4 ; 58,8$ e $67,7 \%$ de mortalidade sobre adultos de $\boldsymbol{S}$. zeamais após sete meses de armazenamento (DEMISSIE et al., 2008).

A redução da emergência de $\boldsymbol{S}$. zeamais pode estar relacionada ao efeito tóxico dos óleos ao embrião do inseto, em virtude da privação de oxigênio (SCHOONHOVEN, 1978), mas é de se esperar que essa toxicidade seja reduzida com o tempo, devido a sua baixa persistência (ISMAN, 2006). Essa informação, em relação à redução da emergência com o prolongamento do período de armazenamento, foi comprovada em pesquisas desenvolvidas por diversos autores (COITINHO et al., 2006; ASAWALAM \& HASSANALI, 2006; DEMISSIE et al., 2008). Essa propriedade pode limitar o uso de

Tabela 2 - Número (média \pm EP) de Sitophilus zeamais emergidos em milho tratado com óleos vegetais, em diferentes concentrações, após a impregnação e aos $30,60,90$ e 120 dias de armazenamento. Temp.: $27,4 \pm 0,91^{\circ} \mathrm{C}$, UR: $62,5 \pm 2,33 \%$ e fotofase de $12 \mathrm{~h}$.

\begin{tabular}{|c|c|c|c|}
\hline Tratamento & {[]$^{3} \mu \mathrm{L} 40 \mathrm{~g}^{-1}$} & Equação de regressão ${ }^{4}$ & Média Geral ${ }^{6,7}$ \\
\hline Testemunha & 0 & $\mathrm{Y}=40,10^{5}$ & $40,1 \pm 1,18 \mathrm{a}$ \\
\hline $\begin{array}{l}\text { Cinnamomum } \\
\text { zeylanicum }{ }^{1}\end{array}$ & 30 & $\begin{array}{l}\mathrm{Y}=4,19+19,19 \mathrm{t}-2,80 \mathrm{t}^{2} \\
\mathrm{R}^{2}=0,72 ; \mathrm{P}<0,0001\end{array}$ & $25,8 \pm 3,31 \mathrm{~b}$ \\
\hline Eugenol & 19 & $\begin{array}{l}Y=3,08+22,42 t-3,91 t^{2} \\
R^{2}=0,53 ; P<0,0016\end{array}$ & $24,5 \pm 3,65 b$ \\
\hline Piper hispidinervum $^{1}$ & 2,8 & $\begin{array}{l}Y=12,7+5,18 t \\
\mathrm{R}^{2}=0,53 ; \mathrm{P}<0,0003\end{array}$ & $23,1 \pm 2,31 \mathrm{~b}$ \\
\hline Schinus terebinthifolius ${ }^{1}$ & 80 & $Y=21,75^{5}$ & $21,8 \pm 1,30 \mathrm{~b}$ \\
\hline Schinus terebinthifolius ${ }^{2}$ & 125 & $\begin{array}{l}\mathrm{Y}=0,67+37,77 \mathrm{t}-16,21 \mathrm{t}^{2}+2,08 \mathrm{t}^{3} \\
\mathrm{R}^{2}=0,77 ; \mathrm{P}<0,0001\end{array}$ & $20,6 \pm 2,63 b$ \\
\hline Melaleuca leucadendron ${ }^{1}$ & 120 & $\begin{array}{l}\mathrm{Y}=0,10+10,00 \mathrm{t} \\
\mathrm{R}^{2}=0,84 ; \mathrm{P}<0,0001\end{array}$ & $20,1 \pm 3,54 b$ \\
\hline Eugenia uniflora $^{1}$ & 35 & $\begin{array}{l}Y=0,55+27,34 t-12,59 t^{2}+1,77 t^{3} \\
R^{2}=0,81 ; P<0,0001\end{array}$ & $15,1 \pm 1,90 \mathrm{~b}$ \\
\hline Piper marginatum $^{1}$ & 40 & $\mathrm{Y}=0,30^{5}$ & $0,30 \pm 0,15 \mathrm{c}$ \\
\hline
\end{tabular}

${ }^{1}$ Folhas; ${ }^{2}$ Frutos Verdes; ${ }^{3}$ Concentração; ${ }^{4}$ Equação de regressão ajustada para número de adultos emergidos nos períodos após a impregnação a 120 dias de armazenamento; ${ }^{5}$ Efeito residual (tempo após tratamento) não significativo; ${ }^{6}$ Dados transformados em $(\mathrm{X}+1)^{0,5} ;{ }^{7} \mathrm{Médias}$ seguidas da mesma letra na vertical não diferem estatisticamente pelo teste de Tukey HSD $(\mathrm{P}>0,05)$.

Ciência Rural, v.40, n.7, jul, 2010. 
óleos essenciais por períodos prolongados de armazenamento.

O modo de ação neurotóxico dos óleos essenciais está relacionado com a rapidez no controle de pragas. Há evidências sobre a interferência no neuromodulador octopamina, que é encontrado em todos os invertebrados, mas não nos mamíferos ou nos canais de cálcio modulados pelo GABA (ISMAN, 2006). A octopamina é semelhante à noradrenalina $\mathrm{e}$ age como neurohormônio, neuromodulador e neurotransmissor, regulando os batimentos cardíacos, os movimentos, o comportamento e o metabolismo dos insetos (ROEDER, 1999).

Os resultados obtidos neste trabalho indicam que o óleo essencial de P. marginatum foi o mais persistente em grãos de milho. Desse modo, este pode ser considerado promissor para o manejo de $\boldsymbol{S}$. zeamais em milho armazenado, principalmente para os agricultores de cultivos familiares e de produção orgânica, pelo fato de a liberação desses compostos ser mais fácil de ser obtida, em relação ao registro de inseticidas sintéticos, aliada às suas vantagens, como eficiência, degradabilidade e segurança para os aplicadores e consumidores de grãos.

\section{CONCLUSÃO}

Os óleos essenciais testados, com exceção de $\boldsymbol{P}$. marginatum, têm baixa persistência em grãos de milho. Por sua vez, esse óleo exerce proteção eficiente às sementes de milho contra o ataque do gorgulho do milho, S. zeamais, durante 120 dias de armazenamento.

\section{AGRADECIMENTOS}

À FACEPE e ao CNPq, pelas bolsas concedidas ao primeiro e ao segundo autor. Ao Prof. Jorge Braz Torres, pelas sugestões, e à Alicely Araújo Correia, a Alberto Belo Esteves Filho e à Solange Maria de França, pelos auxílios prestados.

\section{INFORME VERBAL}

Cláudio Augusto Gomes da Câmara, Endereço Profissional: Universidade Federal Rural de Pernambuco (UFRPE), Departamento de Química, Av. Dom Manoel de Medeiros s/n, 52171-900 - Recife, PE - Brasil. Telefone (81) 33206381. E-mail: camara@dq.ufrpe.br.

\section{REFERÊNCIAS}

ASAWALAM, E.F.; HASSANALI, A. Constituents of the essential oil of Vernonia amygdalina as maize weevil protectants. Tropical and Subtropical Agroecosystems, v.6, n.2, p.95-102, 2006. Disponível em: < http:// www.veterinaria.uady. $\mathrm{mx} /$ publicaciones/journal/2006-2/95vernonia.pdf>. Acesso em: 09 dez. 2008.

COITINHO, R.L.B.C. et al. Efeito residual de inseticidas naturais no controle de Sitophilus zeamais Mots. em milho armazenado. Revista Caatinga, v.19, n.2, p.183-191, 2006. Disponível em: <http://periodicos.ufersa.edu.br/index.php/ sistema/article/view/39/36>. Acesso em: 06 out. 2007.

DEMISSIE, G. et al. Cooking oils and "Triplex" in the control of Sitophilus zeamais Motschulsky (Coleoptera: Curculionidae). Journal of Stored Products Research, v.44, n.2, p.173-178, 2008. Disponível em: <http://dx.doi.org/ 10.1016/j.jspr.2007.10.002.>. Acesso em: 05 jan. 2009. doi:10.1016/j.jspr.2007.10.002.

FAZOLIN, M. et al. Propriedade inseticida dos óleos essenciais de Piper hispidinervum C.DC.; Piper aduncum L. e Tanaecium nocturnum (Barb. Rodr.) Bur. \& K. Schum sobre Tenebrio molitor L., 1758. Ciência e Agrotecnologia, v.31, n.1, p.113-120, 2007. Disponível em: <http://www.scielo.br/pdf/ cagro/v31n1/v31n1a17.pdf>. Acesso em: 23 jan. 2008 . doi: 10.1590/S1413-70542007000100017.

ISMAN, M.B. Botanical insecticides, deterrents, and repellents in modern agriculture and an increasingly regulated world. Annual Review of Entomology, v.51, n.1, p.45-66, 2006. Disponível em: <http://arjournals.annualreviews.org/doi/full/ 10.1146/annurev.ento.51.110104.151146>. Acesso em: 02 fev. 2009. doi:10.1146/annurev.ento.51.110104.151146.

KETOH, G.K. et al. Comparative effects of Cymbopogon schoenanthus essential oil and piperitone on Callosobruchus maculatus development. Fitoterapia, v.77, n.7-8, p.506510, 2006. Disponível em: <http://dx.doi.org/10.1016/ j.fitote.2006.05.031>. Acesso em: 25 out. 2008. doi:10.1016/ j.fitote.2006.05.031.

MAIA, J.G.S. et al. Constituents of the essential oil of Piper aduncum L. growing wild in the Amazon region. Flavour and Fragrance Journal, v.13, n.4, p.269-272, 1998. Disponível em: <http://dx.doi.org/10.1002/(sici)10991026(1998070)13:4<269::aid-ffj744>3.0.co;2-a.>. Acesso em: 14 jan. 2008. doi: 10.1002/(sici)10991026(1998070)13:4<269::aid-ffj744>3.0.co;2-a.

MOREIRA, D.M. et al. Plant compounds insecticide activity against coleoptera pests of stored products. Pesquisa Agropecuária Brasileira, v.42, n.7, p. 909-915, 2007. Disponível em: <http://www.scielo.br/pdf/pab/v42n7/01.pdf>. Acesso em: maio, 2008. doi: 10.1590/S0100204X2007000700001.

RAJENDRAN, S.; SRIRANJINI, V. Plant products as fumigants for stored-product insect control. Journal of Stored Products Research, v.44, n.2, p.126-135, 2008. Disponível em: <http://dx.doi.org/10.1016/j.jspr.2007.08.003>. Acesso em: 06 dez. 2008. doi:10.1016/j.jspr.2007.08.003.

ROEDER, T. "Octopamine in invertebrates". Progress in Neurobiology, v.59, n.5, p.533-561, 1999. Disponível em: $<$ http://dx.doi.org /10.1016/S0301-0082(99)00016-7>. Acesso em: 08 abr. 2008. doi:10.1016/S0301-0082(99)00016-7.

SAHAF, B.Z.; MOHARRAMIPOUR, S. Fumigant toxicity of Carum copticum and Vitex pseudo-negundo essential oils against eggs, larvae and adults of Callosobruchus maculatus. Journal of Pest Science, v.81, n.4, p.213-220, 2008. Disponível em: <http://www.springerlink.com/content/ axh2t7680xx41667/fulltext.pdf>. Acesso em: 20 dez. 2008. doi: $10.1007 / \mathrm{s} 10340-008-0208-y$.

SAS INSTITUTE. SAS/Stat user's guide. Cary, 2002. 1 CD.

SCHOONHOVEN, A.V. Use of vegetable oils to protect store bean from bruchid attack. Journal of Economic Entomology, v.71, n.1, p.254-256, 1978. 